\title{
Metastatic Small-Cell Neuroendocrine Carcinoma Simulating Circumscribed Choroidal Hemangioma
}

\author{
Kate E. Leahy ${ }^{a}$ Tanya Karaconji ${ }^{a, c}$ Valli Thanni ${ }^{b}$ Anita Achan ${ }^{b}$ \\ Adrian T. Fung ${ }^{\mathrm{c}-\mathrm{e}}$ \\ Departments of a Ophthalmology and ${ }^{b}$ Anatomical Pathology, Institute for Clinical Pathology and Medical Research, \\ Westmead Hospital, ' Sydney Eye Hospital, d Save Sight Institute, University of Sydney, and e Australian School of \\ Advanced Medicine, Macquarie University, Sydney, N.S.W., Australia
}

\section{Key Words}

Choroidal metastasis - Immunohistochemistry - Metastasis . Tumor - Small-cell carcinoma - Neuroendocrine carcinoma . Choroidal hemangioma

\section{Abstract}

Aim: To report a case of metastatic small-cell neuroendocrine carcinoma presenting as an isolated choroidal mass and initially misdiagnosed as a circumscribed choroidal hemangioma. Methods: The clinical history, fundus findings, imaging, cytology and immunohistochemical features are described. Results: An otherwise healthy 66-year-old man was referred for a left nasal scotoma and a diagnosis of circumscribed choroidal hemangioma. Cytology showed cohesive clusters of small-to-intermediate malignant cells. The atypical cells stained positively for chromogranin, thyroid transcription factor-1 and synaptophysin consistent with small-cell neuroendocrine carcinoma. Conclusion: Smallcell neuroendocrine carcinoma metastatic to the choroid is extremely rare; however, it is particularly aggressive and should be included in the differential diagnosis of isolated choroidal lesions, even in otherwise healthy patients.

(c) 2015 S. Karger AG, Basel

\section{Introduction}

We describe a case of metastatic neuroendocrine carcinoma presenting as an isolated choroidal mass and initially misdiagnosed as a circumscribed choroidal hemangioma.

\section{Case Report}

A 66-year-old Caucasian man was referred with a left nasal scotoma and a diagnosis of circumscribed choroidal hemangioma. Past history included an extensive right facial mass present since birth consistent with a capillary malformation involving the V2 dermatome (fig. 1a). He was an otherwise healthy nonsmoker with no history of malignancy.

Best-corrected visual acuities were $6 / 6$ in the right eye and 6/24 in the left eye. A raised, orange, nonpigmented choroidal mass presented just temporal to the left fovea with associated exudative retinal detachment (fig. 1b). The choroidal lesion was hyperautofluorescent and hypofluorescent on fundus fluorescein angiography and indocyanine green angiography (fig. 1c-e). On B-scan ultrasonography, it was echodense and measured $6.0 \mathrm{~mm}$ in height and $16.3 \mathrm{~mm}$ in its greatest basal dimension (fig. 1f). MRI showed avid enhancement of the lesion on postgadolinium T1 sequences (fig. $1 \mathrm{~g}$ ). Brain windows revealed multiple lesions consistent with metastases.

\section{KARGER}

E-Mail karger@karger.com

www.karger.com/oop (c) 2015 S. Karger AG, Base

2296-4681/15/0021-0013\$39.50/0
Dr. Adrian T. Fung

Retina \& Macula Specialists

$1 \mathrm{~A} / 12$ Central Road

Miranda, NSW 2228 (Australia)

E-Mail adrianfungi@yahoo.com.au 
Two weeks after initial presentation, the patient developed testicular swelling and abdominal pain secondary to ascites. CT of the chest, abdomen and pelvis demonstrated multiple metastases involving the mediastinum, lungs, adrenals and kidneys.

A liver biopsy and ascitic, perihepatic and pleural fluid samples were analyzed. Cytologic evaluation and the immunohistochemis-
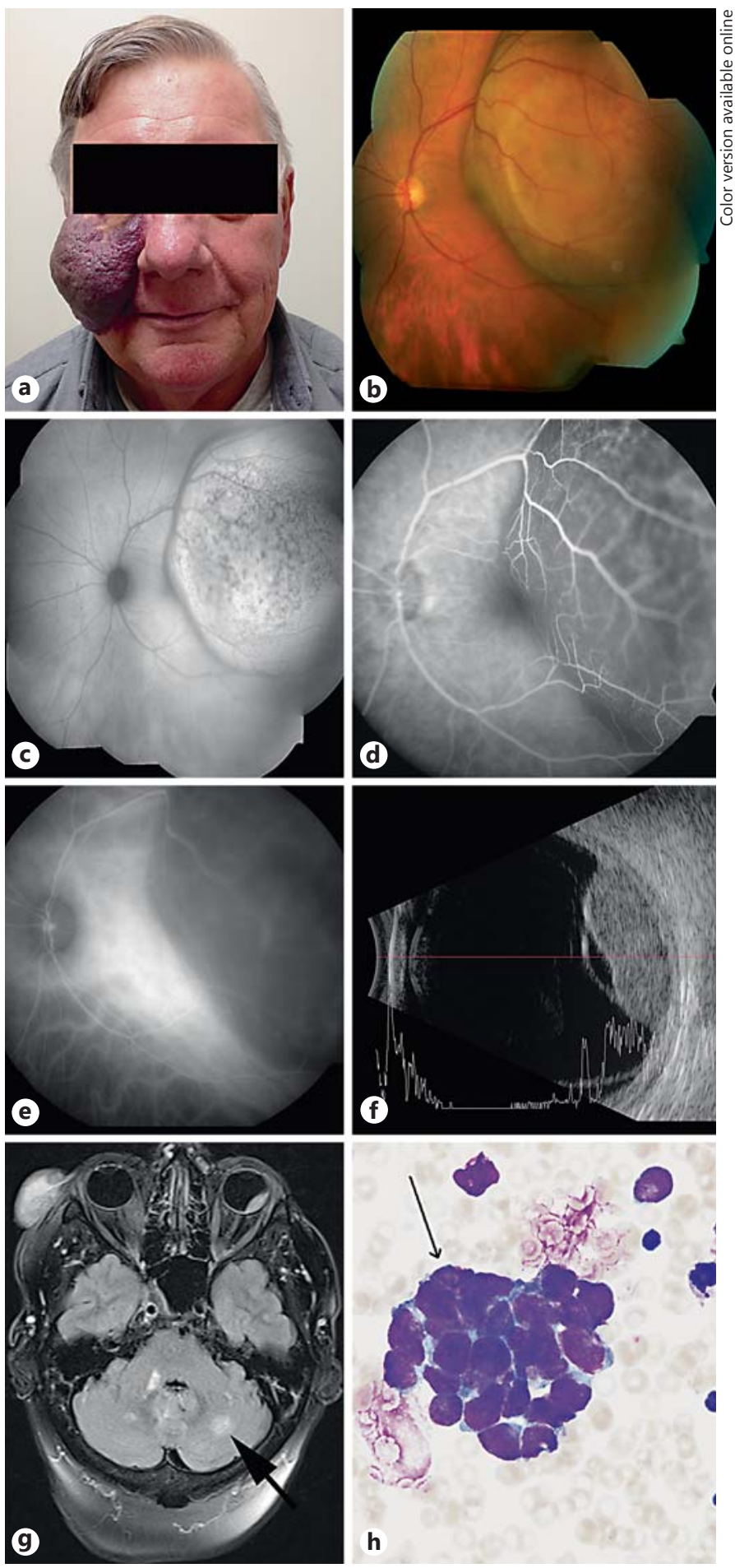

try profile were consistent with small-cell neuroendocrine carcinoma (fig. 1h). The atypical cells stained positively for chromogranin, thyroid transcription factor-1 and synaptophysin. The choroidal lesion was therefore presumed to represent a metastasis of small-cell neuroendocrine carcinoma. Carboplatin and etoposide chemotherapy was commenced. During the first cycle, the patient developed acute renal injury and tumor lysis syndrome. $\mathrm{He}$ passed away 2 months later with a diagnosis of small-cell neuroendocrine carcinoma of probable lung primary.

\section{Discussion}

The differential diagnoses of this choroidal lesion included choroidal hemangioma, amelanotic choroidal melanoma and choroidal metastasis. Choroidal hemangioma was initially considered by the referring ophthalmologist because of the orange color of the lesion and the echodensity on B-scan ultrasonography. Although facial capillary malformations can be associated with SturgeWeber syndrome and this, in turn, with choroidal hemangioma, they are nearly always diffuse and ipsilateral [1]. In addition, choroidal hemangiomas are usually hyperfluorescent on fundus fluorescein angiography and indocyanine green. Choroidal melanoma was less likely given the nonpigmented orange color and echodensity on Bscan ultrasonography.

Neuroendocrine carcinoma metastatic to the choroid is extremely rare [2-4]. We are aware of only 3 prior case reports, all of which differ from ours in that the lesions were pigmented [2], multiple and bilateral [3] or associated with a known primary carcinoma [4]. Most choroidal metastases are unifocal, and in one third of the cases, there is no known history of cancer at presentation [5]. Smallcell neuroendocrine carcinoma is a particularly aggressive form of neuroendocrine carcinoma noted for its rapid and early metastatic dissemination. Like choroidal metastases in general, it most commonly arises from the lungs $[3,5]$.

Fig. 1. a, b A 66-year-old Caucasian man presented with a right facial capillary malformation and a left orange-colored choroidal mass. c-e The lesion is hyperautofluorescent and hypofluorescent on fluorescein indocyanine green angiography. $f$ On B-scan ultrasound, the lesion is bilobed, echodense and measures $6.0 \mathrm{~mm}$ in apical height and $16.3 \mathrm{~mm}$ in its greatest basal dimension. Associated serous retinal detachment is present overlying the lesion and inferiorly. g T1-weighted MRI shows gadolinium enhancement of the lesion and multiple cerebral metastases (thick arrow). $\mathbf{h}$ DiffQuik stain (original magnification $\times 400$ ) of ascitic fluid cytology shows small-to-intermediate malignant cells arranged in cohesive clusters with nuclear molding (thin arrow). 
This case emphasizes that metastatic neuroendocrine carcinoma should be included in the differential diagnosis of isolated choroidal lesions, even in otherwise healthy patients.

\section{Acknowledgements} lication.

The patient consented to the use of his photograph in this pub-

Dr. Raghwa Sharma MBBS, FRCPA, Institute for Clinical Pathology and Medical Research, Westmead Hospital, provided diagnostic advice.

\section{Statement of Ethics}

We have no ethical conflicts to disclose.

\section{Disclosure Statement}

The authors declare that there are no conflicts of interest to disclose.

\section{References}

-1 Tsipursky MS, Golchet PR, Jampol LM: Photodynamic therapy of choroidal hemangioma in Sturge-Weber syndrome, with a review of treatments for diffuse and circumscribed choroidal hemangiomas. Surv Ophthalmol 2011; 56:68-85.

-2 Eagle RC Jr, Ehya H, Shields JA, Shields CL: Choroidal metastasis as the initial manifestation of a pigmented neuroendocrine tumor. Arch Ophthalmol 2000;118:841-845.
3 Nicolo M, Piccolino FC, Ghiglione D, Nicolo G, Calabria G: Multiple bilateral choroidal metastatic tumors from a small-cell neuroendocrine carcinoma of unknown primary site. Eur J Ophthalmol 2005;15:148-152.

-4 Krema H, McGowan H, Tanzer H, Simpson $\mathrm{R}$, Laperriere N: Unusual orange-coloured choroidal metastases. J Ophthalmic Vis Res 2013;8:53-57.

5 Shields CL, Shields JA, Gross NE, Schwartz GP, Lally SE: Survey of 520 eyes with uveal metastases. Ophthalmology 1997;104:12651276. 